\title{
Optimizing the structural topology of bifunctional invisible cloak manipulating heat flux and direct current
}

Cite as: Appl. Phys. Lett. 115, 174101 (2019); https://doi.org/10.1063/1.5123908

Submitted: 09 August 2019 • Accepted: 02 October 2019 • Published Online: 21 October 2019

(D) Garuda Fujii and (D) Youhei Akimoto
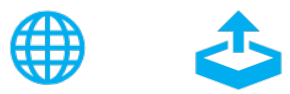

View Online

\section{ARTICLES YOU MAY BE INTERESTED IN}

Exploring optimal topology of thermal cloaks by CMA-ES

Applied Physics Letters 112, 061108 (2018); https://doi.org/10.1063/1.5016090

Shaped graded materials with an apparent negative thermal conductivity Applied Physics Letters 92, 251907 (2008); https://doi.org/10.1063/1.2951600

Gradient-index phononic crystals for highly dense flexural energy harvesting Applied Physics Letters 115, 173901 (2019); https://doi.org/10.1063/1.5111566

\section{Challenge us.} What are your needs for periodic signal detection?

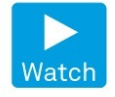

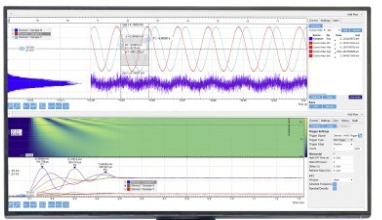

0
Zurich Instruments 


\title{
Optimizing the structural topology of bifunctional invisible cloak manipulating heat flux and direct current
}

Cite as: Appl. Phys. Lett. 115, 174101 (2019); doi: 10.1063/1.5123908

Submitted: 9 August 2019 - Accepted: 2 October 2019 •

Published Online: 21 October 2019

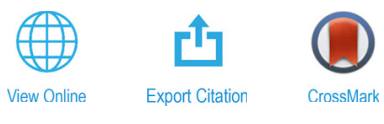

Garuda Fujii $^{1, a)}$ (D) and Youhei Akimoto ${ }^{2}$

\begin{abstract}
AFFILIATIONS
Institute of Engineering, Shinshu University, 4-17-1 Wakasato, Nagano City 380-8553, Japan

${ }^{2}$ Center for Artificial Intelligence Research, University of Tsukuba, 1-7-1 Tennodai, Tsukuba 305-8573, Japan
\end{abstract}

a) Author to whom correspondence should be addressed: g_fujii@shinshu-u.ac.jp

\begin{abstract}
We generate optimal topologies in the structural design of bifunctional cloaks manipulating heat flux and direct current, using topology optimization that incorporates both thermal conductivity and electrical direct current. The bifunctional cloak composed of bulk isotropic materials is designed to restrain thermal and electrical disturbances caused by an insulated obstacle by minimizing the difference between cloaked distributions and referenced distributions when no obstacle is present. Our results show that the presented optimizations provide bifunctional cloaks that reproduce undisturbed temperature and voltage distributions. We also demonstrate topology optimizations for bifunctional cloaks operating for multiangle flows and those for cloaks that are robust against variations in conductive properties.
\end{abstract}

Published under license by AIP Publishing. https://doi.org/10.1063/1.5123908

Optical invisibility ${ }^{1}$ has been an enthusiastically pursued field of research since the proposals of transformation optics. ${ }^{2,3}$ The coordinate transformation approach is the most adopted theoretical method of realizing invisibility, camouflage, and illusion and has spread to various fields of physics, such as acoustics, ${ }^{4}$ thermodynamics, ${ }^{5}$ and electrical engineering. ${ }^{6}$ This has led to several innovations, such as invisible sensors ${ }^{7,8}$ and camouflage of heat signatures.

Topology optimization ${ }^{10}$ has recently received attention as another way to produce invisibility. Topology optimization is the most flexible numerical method of structural design with the highest level of design freedom and allows for topological changes, including the appearance of holes, during an optimization procedure. This methodology improves device performance drastically, and optimal cloaking structures have been designed in optics, ${ }^{11-16}$ acoustics, ${ }^{17,18}$ thermotics, ${ }^{19,20}$ and electrical engineering. ${ }^{21,22}$ However, these topologyoptimized cloaks exhibit cloaking only for one type of physics (i.e., in the design of unifunctional cloaks), because existing topology optimization approaches for cloaking improve structures only through the analysis of a single physical flow. For instance, topology optimization has not been developed for the design of a bifunctional cloak that realizes invisibility for two different types of physical flow.

Since the pioneering theoretical proposal ${ }^{23}$ for bifunctional cloaks that manipulate both heat flow and electrical direct current (DC), an independent manipulation of heat and electrical current has been demonstrated using bifunctional metamaterials. ${ }^{24}$ However, this approach has structural challenges to overcome because the bifunctional metamaterial has complicated microstructures. To overcome this, several experiments to cloak an object in multiphysical flows have been attempted using a bifunctional shell system ${ }^{25,26}$ and a combination of passive and active schemes. ${ }^{27}$ These approaches originated from the bilayer strategy for heat flow ${ }^{28,29}$ and electric current. ${ }^{30}$ Those devices can be developed without artificial inhomogeneity; specifically, they can be made using only natural materials, simplifying their fabrication.

In this work, we optimized the topology of thermal-electrical bifunctional cloaks made of copper in an iron host. Both the temperature and voltage distributions when no obstacle is present are reproduced using topology-optimized bifunctional cloaks as if nothing exists despite the presence of the obstacle. We employ an immersed boundary-level set method for structural expression, the covariance matrix adaptation evolution strategy (CMA-ES) for exploring the optimal configuration, and a perimeter constraint for the regularization of the ill-posedness.

Figure 1 shows the optimization scheme for the bifunctional cloak. An insulator $\Omega_{\text {ins }}$ is located at the center of a host structure made of iron $(\mathrm{Fe})$, acting as an obstacle disturbing the thermal 
(a)

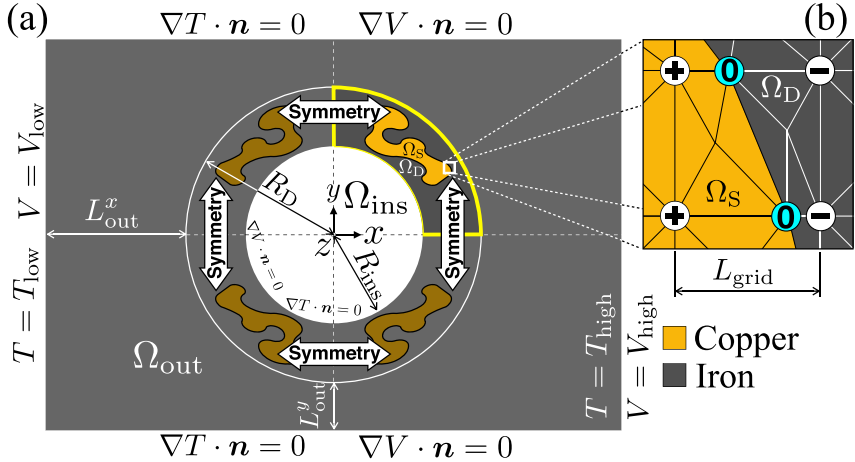

FIG. 1. (a) Schematic illustration of topology optimization for the thermal-electrical bifunctional cloak. Domain sizes are set to $R_{\text {ins }}=3 R_{\mathrm{D}} / 5, L_{\text {out }}^{x}=R_{\mathrm{D}}, L_{\text {out }}^{y}=R_{\mathrm{D}} / 3$, and $L_{\text {grid }}=R_{\mathrm{D}} / 90$. Copper $(\mathrm{Cu})$ and iron $(\mathrm{Fe})$ are assumed to be the materials of the designed and host structure, respectively; their thermal and electrical conductivities are $\kappa_{\mathrm{Cu}}=386 \mathrm{Wm}^{-1} \mathrm{~K}^{-1}, \kappa_{\mathrm{Fe}}=67.0 \mathrm{Wm}^{-1} \mathrm{~K}^{-1}, \sigma_{\mathrm{Cu}}=59.0 \times 10^{6} \mathrm{Sm}^{-1}$, and $\sigma_{\mathrm{Fe}}=9.90 \times 10^{6} \mathrm{Sm}^{-1}$. (b) Discretized level-set functions $\phi_{j}$ on grid points and a structural boundary. The plus and minus symbols represent the signs of $\phi_{j}$ on grid points, and 0 marks the location of a structural boundary obtained from $\phi(x)=0$.

conduction and DC. The fixed design domain $\Omega_{\mathrm{D}}$ is set to cover the insulator, and the designed structure made of copper $(\mathrm{Cu})$ is transformed in $\Omega_{\mathrm{D}}$ with structural symmetries imposed about $x$ and $y$ axes. The outer domain of $\Omega_{\mathrm{D}}$ is denoted $\Omega_{\text {out }}$, where thermal and electrical disturbances are evaluated using the objective functions defined below. Both thermally and electrically insulated boundary conditions are imposed on the upper and lower boundaries of the host structure. A low temperature and low voltage on the left boundary of the host and a high temperature and high voltage on the right boundary are introduced to simulate directed transfer and current for negative $x$.

A thermal-electrical bifunctional cloak is designed to restrain the disturbances of temperature and voltage by reproducing both the temperature and voltage distributions when no insulator is present despite the presence of the insulator. The objective functions for thermal and DC electric cloaks, respectively denoted $\Psi_{T}$ and $\Psi_{V}$, are defined as

$$
\begin{aligned}
& \Psi_{T}=\frac{1}{\Psi_{T}^{\text {bare }}} \int_{\Omega_{\text {out }}}\left|T-T_{\text {ref }}\right|^{2} d \Omega, \\
& \Psi_{V}=\frac{1}{\Psi_{V}^{\text {bare }}} \int_{\Omega_{\text {out }}}\left|V-V_{\text {ref }}\right|^{2} d \Omega,
\end{aligned}
$$

where $T$ and $T_{\text {ref }}$ are, respectively, the temperature field when the insulator is cloaked and the reference temperature field with no disturbance when no insulator is present, and $V$ and $V_{\text {ref }}$ are voltage fields given in a similar manner. The above objective functions are normalized, respectively, by $\Psi_{T}^{\text {bare }}$ and $\Psi_{V}^{\text {bare }}$ and defined as

$$
\begin{aligned}
\Psi_{T}^{\text {bare }} & =\int_{\Omega_{\text {out }}}\left|T_{\text {bare }}-T_{\text {ref }}\right|^{2} d \Omega, \\
\Psi_{V}^{\text {bare }} & =\int_{\Omega_{\text {out }}}\left|V_{\text {bare }}-V_{\text {ref }}\right|^{2} d \Omega,
\end{aligned}
$$

where $T_{\text {bare }}$ and $V_{\text {bare }}$ are, respectively, temperature and voltage fields when a bare insulator is present without a cloak. To design bifunctional cloaks for heat transfer and DC, the fitness minimized in the presented topology optimization is given as

$$
\inf _{\phi} \quad F_{\mathrm{r}}=\left(\Psi_{T}+\Psi_{V}\right) / 2+\tau L_{\mathrm{p}}
$$

where $\phi$ represents the level set function optimized as a design variable, $L_{\mathrm{p}}$ is the perimeter of the structure (i.e., the total length of the boundaries between $\Omega_{\mathrm{S}}$ and $\Omega_{\mathrm{D}} \backslash \Omega_{\mathrm{S}}$ ) normalized by the radius of the fixed design domain, $R_{\mathrm{D}}$, to regularize the ill-posedness of topology optimization, and $\tau$ is the regularization coefficient that determines the ratio between the average of the objective functions and the perimeter. The regularization coefficient $\tau$ is a continuous variable, and a suitable $\tau$ can be set based on the ratio between the target value for $L_{\mathrm{p}}$ and the target values for $\Psi_{T}$ and $\Psi_{V}$. The perimeter $L_{\mathrm{p}}$ and the cloaking performance of the optimal bifunctional cloaks, $\Psi_{T}$ and $\Psi_{V}$, face tradeoffs but can be controlled by adjusting $\tau$. The coefficient is never set to $\tau=0$, because this means no perimeter constraint, causing the topology optimization to become an ill-posed problem.

The temperature and voltage in the steady state follow governing equations referred to as Laplace's equations:

$$
\nabla \cdot(\kappa(\boldsymbol{x}) \nabla T)=0, \quad \nabla \cdot(\sigma(\boldsymbol{x}) \nabla V)=0,
$$

where $\kappa(\boldsymbol{x})$ and $\sigma(\boldsymbol{x})$ are, respectively, the thermal and electrical conductivities, given as

$$
\kappa(\boldsymbol{x})= \begin{cases}\kappa_{\mathrm{Fe}}+\chi\left(\kappa_{\mathrm{Cu}}-\kappa_{\mathrm{Fe}}\right) & \text { for } \boldsymbol{x} \in \Omega_{\mathrm{D}} \\ \kappa_{\mathrm{Fe}} & \text { for } \boldsymbol{x} \in \Omega_{\mathrm{out}},\end{cases}
$$

and

$$
\sigma(\boldsymbol{x})= \begin{cases}\sigma_{\mathrm{Fe}}+\chi\left(\sigma_{\mathrm{Cu}}-\sigma_{\mathrm{Fe}}\right) & \text { for } \boldsymbol{x} \in \Omega_{\mathrm{D}} \\ \sigma_{\mathrm{Fe}} & \text { for } \boldsymbol{x} \in \Omega_{\mathrm{out}},\end{cases}
$$

where $\kappa_{\mathrm{Fe}}$ and $\kappa_{\mathrm{Cu}}$ are the thermal conductivities of iron and copper, respectively, and $\sigma_{\mathrm{Fe}}$ and $\sigma_{\mathrm{Cu}}$ are the electrical conductivities of iron and copper, respectively. Here, $\chi$ is the characteristic function defined as

$$
\chi(\phi(x))= \begin{cases}1 & \text { if } x \in \Omega_{\mathrm{S}} \\ 0 & \text { if } x \in \Omega_{\mathrm{D}} \backslash \Omega_{\mathrm{S}},\end{cases}
$$

where $\phi(\boldsymbol{x})$ is the level set function determining the domains as

$$
\begin{aligned}
\Omega_{\mathrm{S}} \backslash \Gamma_{\mathrm{S}} & =\{\boldsymbol{x} \mid 0<\phi(\boldsymbol{x}) \leq 1\}, \\
\Gamma_{\mathrm{S}} & =\{\boldsymbol{x} \mid \phi(\boldsymbol{x})=0\}, \\
\Omega_{\mathrm{D}} \backslash \Omega_{\mathrm{S}} & =\{\boldsymbol{x} \mid-1 \leq \phi(\boldsymbol{x})<0\} .
\end{aligned}
$$

Here, $\Gamma_{S}$ represents interfaces between copper and iron structures as shown in Fig. 1(b). The level set function $\phi(\boldsymbol{x})$ is discretized into grid points as $\boldsymbol{\phi}=\left\{\phi_{1}, \ldots, \phi_{j}, \ldots, \phi_{n}\right\}$, and the optimal $\phi$ is explored by adopting the CMA-ES ${ }^{31}$ with a box constraint handling. ${ }^{16}$

In numerical demonstrations, temperature and voltage are normalized as $0 \leq T \leq 1$ and $0 \leq V \leq 1$, respectively, by using the highest and lowest values of those quantities: $T_{\text {high }}, T_{\text {low }}, V_{\text {high }}$, and $V_{\text {low }}$. The thermal and electrical conductivities are also normalized by those of the host material: $\kappa_{\mathrm{Fe}}$ and $\sigma_{\mathrm{Fe}}$, respectively.

The structure in the fixed design domain $\Omega_{\mathrm{D}}$ without an insulator is presented in Fig. 2(a). Copper and insulated structures are not presented, and the system is uniformly composed of iron. The corresponding temperature and voltage are distributed with a constant gradient as, respectively, shown in Figs. 2(b) and 2(d), and heat flux 


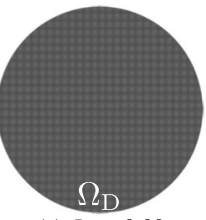
(a) $L_{\mathrm{p}}=0.00$

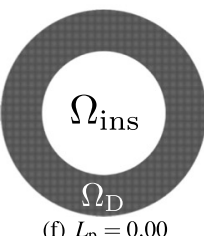

(f) $L_{\mathrm{p}}=0.00$

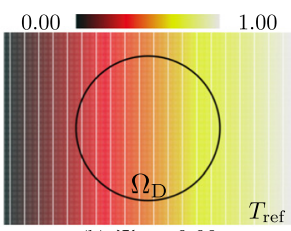

(b) $\Psi_{T}=0.00$

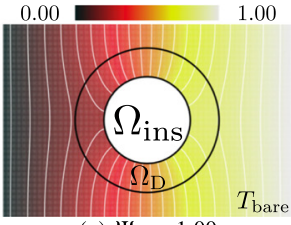

(g) $\Psi_{T}=1.00$

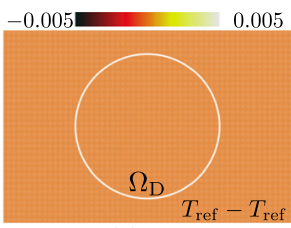

(c) $\Psi_{T}=0.00$

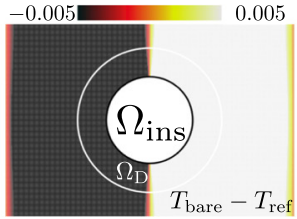

(h) $\Psi_{T}=1.00$

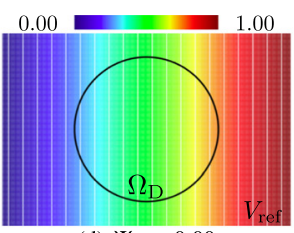

(d) $\Psi_{V}=0.00$

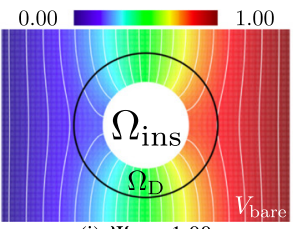

(i) $\Psi_{V}=1.00$

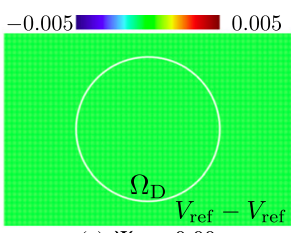

(e) $\Psi_{V}=0.00$

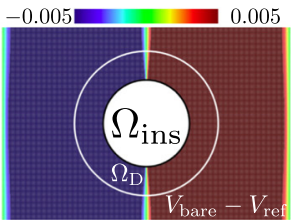

(j) $\Psi_{V}=1.00$

FIG. 2. Analysis results (a)-(e) when no insulator is present and ( $f$ - $-(j)$ when an insulator is present without a cloak.

and DC are directed in the negative $x$ direction. Contour lines of the distributions are straight, and the distributions are the objectives reproduced by the bifunctional cloak.

Figure 2(f) shows the bare insulator located at the center of $\Omega_{D}$ without a copper structure for cloaking. When the insulator is present, the temperature and voltage distributions in Figs. 2(g) and 2(i), respectively, show $T_{\text {bare }}$ and $V_{\text {bare }}$. The distributions are obviously disturbed, and the described contour lines bend around the insulator. The values of the objective functions, $\Psi_{T}$ and $\Psi_{V}$, become 1 in the normalizations. To emphasize the disturbances of the temperature and voltage by the insulator, we plot the evaluated differences, $T-T_{\text {ref }}$ and $V-V_{\text {ref }}$, in Figs. 2(c), 2(e), 2(h), and 2(j) in the fine ranges -0.005 $\leq T-T_{\text {ref }} \leq 0.005$ and $-0.005 \leq V-V_{\text {ref }} \leq 0.005$.

Figure 3 shows the results of the demonstrated topology optimization for the bifunctional cloak. The regularization coefficient of the perimeter constraint is set to $\tau=0.01,0.001$, and 0.0001 . When the coefficient is set larger to $\tau=0.01$, the perimeter constraint is strongly imposed and the obtained optimal configuration becomes simpler with a smaller structural perimeter $L_{\mathrm{p}}=3.83$, as shown in Fig. 3(a). The "eyelid-shaped" optimal configuration in Fig. 3(a) is considered to be indispensable for bifunctional cloaking because the structure is obtained under the stronger constraint. Meanwhile, when a weaker perimeter constraint is imposed as $\tau=0.0001$, the optimal configuration in Fig. $3(\mathrm{k})$ is allowed to be more complex with a longer structural perimeter $L_{\mathrm{p}}=12.2$, and the cloaking performance is enhanced as $\Psi_{T}=0.000142$ and $\Psi_{V}=0.000122$, as shown in Figs. 3(1) and 3(n). In the outer domain $\Omega_{\text {out }}$, the contour lines of temperature and voltage distributions become almost completely straight and there is little disturbance in the evaluated differences shown in Figs. 3(m) and 3(o) despite the plots having a fine range. The cloaked insulator showed

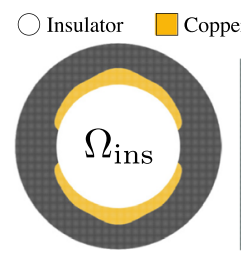

(a) $L_{\mathrm{p}}=3.83$

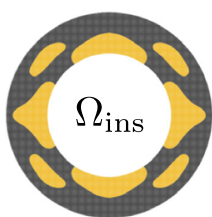

(f) $L_{\mathrm{p}}=8.49$

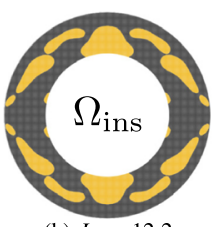

(k) $L_{\mathrm{p}}=12.2$

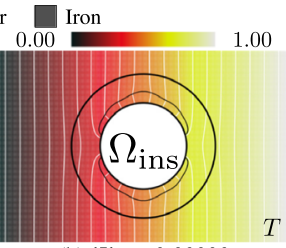

(b) $\Psi_{T}=0.00290$

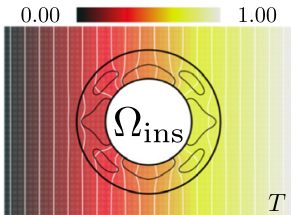

(g) $\Psi_{T}=0.000447$

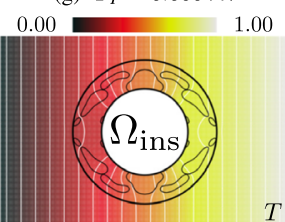

(l) $\Psi_{T}=0.000142$

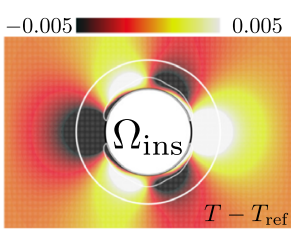

(c) $\Psi_{T}=0.00290$

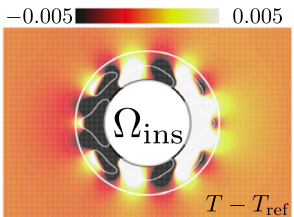

(h) $\Psi_{T}=0.000447$

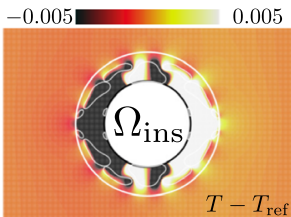

(m) $\Psi_{T}=0.000142$

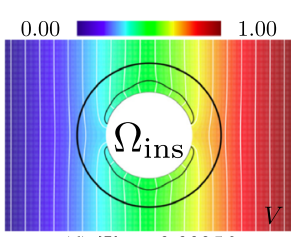

(d) $\Psi_{V}=0.00256$

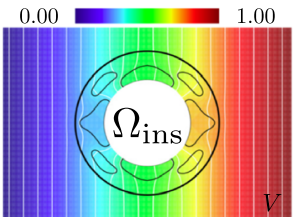

(i) $\Psi_{V}=0.000353$

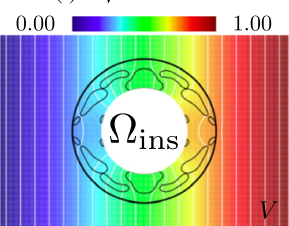

(n) $\Psi_{V}=0.000122$

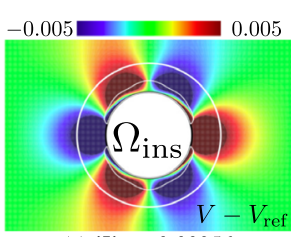

(e) $\Psi_{V}=0.00256$

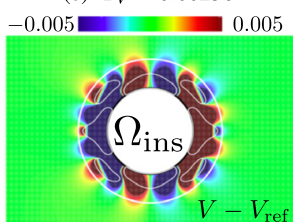

(j) $\Psi_{V}=0.000353$

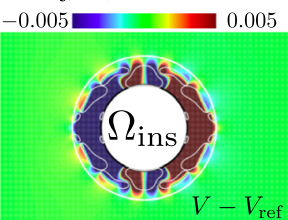

(o) $\Psi_{V}=0.000122$

FIG. 3. Optimization results obtained for (a)-(e) $\tau=0.01$, (f)-(j) $\tau=0.001$, and (k)-(o) $\tau=0.0001$. Optimal configurations (a), (f), and (k) are composed of copper (orange) and iron (dark gray). The fitness values are (a) $F_{\mathrm{r}}=0.0410$, (f) $F_{\mathrm{r}}=0.00889$, and (k) $F_{\mathrm{r}}=0.00135$. The number of candidate solutions is set to $n_{\mathrm{cs}}=80$. The number of design variables becomes $n=4113$. 


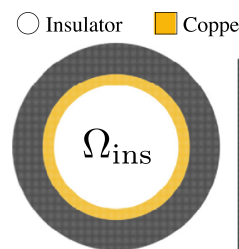

(a) $L_{\mathrm{p}}=4.47$

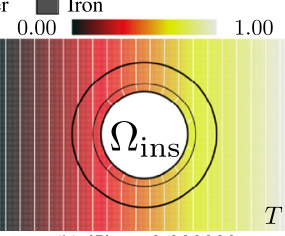

(b) $\Psi_{T}=0.000322$

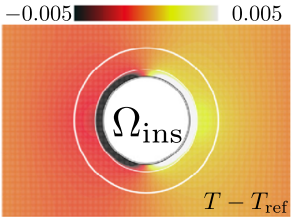

(c) $\Psi_{T}=0.000322$

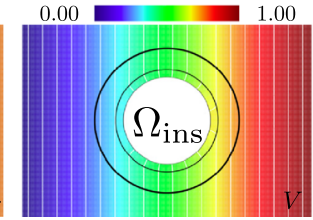

(d) $\Psi_{V}=0.0000498$

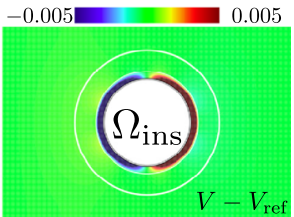

(e) $\Psi_{V}=0.0000498$

FIG. 4. Optimization results obtained under structural symmetries about not only $x$ and $y$ axes but also $y= \pm x$ for $\tau=0.01$. (a) Optimal configuration obtained for $\tau=0.01$, (b) corresponding $T$, and (c) $T-T_{\text {ref, }}$ (d) $V$, and (e) $V-V_{\text {ref }}$ are shown. Fitness value becomes $F_{\mathrm{r}}=0.0449$. The number of candidate solutions is set to $n_{\mathrm{cs}}=80$. The number of design variables becomes $n=2069$.

few disturbances in the two physical flows for being "undetectable," and the optimal configurations made the insulator almost completely "invisible" in both thermal flow and electrical direct current.

Note that it takes about $120 \mathrm{~h}$ to obtain the results in Fig. 3(a) when we use computers with 40 cores. The computation time depends on the specifications of machinery and the discretization denoted by the grid size, $L_{\text {grid }}$. To investigate how discretization affects the optimization result and computation time, we demonstrated topology optimization under various grid sizes $-L_{\text {grid }}=R_{\mathrm{D}} / 15(n=122)$, $R_{\mathrm{D}} / 30(n=467), R_{\mathrm{D}} / 45(n=1038), R_{\mathrm{D}} / 60(n=1835)$, and $R_{\mathrm{D}} /$ $75(n=2860)$-with the regularization coefficient $\tau=0.01$. Except for $L_{\text {grid }}=R_{\mathrm{D}} / 15$, the "eyelid-shaped" optimal configurations are commonly obtained under different $L_{\text {grid }}$. For $L_{\text {grid }}=R_{\mathrm{D}} / 15$, discretization is coarse and finite element analyses become inaccurate in representing the underlying physics. The computation time needed for optimization becomes about $2 \mathrm{~min}\left(L_{\text {grid }}=R_{\mathrm{D}} / 15\right), 28 \mathrm{~min}$ $\left(L_{\text {grid }}=R_{\mathrm{D}} / 30\right), 8 \mathrm{~h}\left(L_{\text {grid }}=R_{\mathrm{D}} / 45\right), 24 \mathrm{~h}\left(L_{\text {grid }}=R_{\mathrm{D}} / 60\right)$, and $66 \mathrm{~h}$ ( $L_{\text {grid }}=R_{\mathrm{D}} / 75$ ) to satisfy a convergence criterion. ${ }^{16}$ If the designer is concerned about calculation time, set $L_{\text {grid }}=R_{\mathrm{D}} / 30$. On the other hand, if importance is placed on calculation accuracy, $L_{\text {grid }}$ is set to smaller because the calculation accuracy of numerical analysis is improved by finer discretization.

To increase the angle of bifunctional invisibility, we demonstrate topology optimization under structural symmetries about not only the $x$ and $y$ axes but also $y= \pm x$. Figure 4 shows the demonstrated optimization. Interestingly, the obtained optimal configuration in Fig. 4(a) becomes almost rotationally symmetric even though rotational symmetry is not imposed. The rotationally symmetric structure corresponds well to bilayer structures ${ }^{28-30}$ by understanding the insulator as the inner polystyrene and by placing another, smaller aluminum cylinder in the insulated domain $\Omega_{\text {ins }}$ as an alternative cloaked object. The optimization result indicates that the bilayer cloaks are among the optimal structures for bifunctional cloaking under the $x, y$, and $y= \pm x$ axial symmetries. In other words, topology optimization does not become advantageous to the bilayer strategy for cloaking the cylindrical insulator. For more complex design problems, such as cloaking irregularly shaped insulating regions, ${ }^{32,33}$ topology optimization would be a better or unique choice because of its design flexibility. A robust design that considers material conductivity is one such design problem and is described later.

Figure 5 shows the relation between the normalized thermal conductivity $\kappa_{\mathrm{S}} / \kappa_{\text {host }}$ and the objective functional value for thermal cloaking $\Psi_{T}$ and that between the normalized electrical conductivity $\sigma_{\mathrm{S}} / \sigma_{\text {host }}$ and the objective functional value for DC electrical cloaking $\Psi_{V}$. The dependence of $\Psi_{T}$ on $\kappa_{\mathrm{S}} / \kappa_{\text {host }}$ exactly corresponds to that of
$\Psi_{V}$ on $\sigma_{\mathrm{S}} / \sigma_{\text {host }}$ because the governing equation and boundary conditions are numerically identical to each other. The dips in the two objective functions are located between horizontal-axis values of 5.76 and 5.96. When we compare the cloaking performances $\Psi_{T}$ and $\Psi_{V}$ for a vertical-axis value of 0.01 , which means that the cloaked disturbances are restrained to $1 \%$ of the disturbance generated by a bare insulator, the performance standards $\Psi_{T} \leq 0.01$ and $\Psi_{V} \leq 0.01$ are satisfied at horizontal-axis values between 5.22 and 6.74 for $\tau=0.01$, 4.96, and 7.04 for $\tau=0.001$, and 4.96 and 6.95 for $\tau=0.0001$.

To improve the robustness of the cloaking performance against relative conductivities, we numerically demonstrate topology optimizations minimizing the alternative fitness function

$$
\inf _{\phi} \quad F_{\max }=\Psi_{\max }+\tau L_{\mathrm{p}}
$$

Here, $\Psi_{\max }$ denotes the maximum values of $\Psi_{T}$ under $\kappa_{\mathrm{S}} / \kappa_{\text {host }}=4$, 6 , and 8 for thermal cloaking and those of $\Psi_{V}$ under $\sigma_{\mathrm{S}} / \sigma_{\text {host }}=4,6$, and 8 for DC electric cloaking and is given as

$$
\Psi_{\max }=\max \left(\Psi_{T}^{4}, \Psi_{T}^{6}, \Psi_{T}^{8}, \Psi_{V}^{4}, \Psi_{V}^{6}, \Psi_{V}^{8}\right),
$$

where $\Psi_{T}^{\kappa_{\mathrm{S}} / \kappa_{\text {host }}}$ is the objective function under the relative thermal conductivity $\kappa_{\mathrm{S}} / \kappa_{\text {host }}$, while $\Psi_{V}^{\sigma_{\mathrm{S}} / \sigma_{\text {host }}}$ is that under $\sigma_{\mathrm{S}} / \sigma_{\text {host }}$.

Optimal configurations obtained by minimizing $\Psi_{\max }$ and the robustness of the cloaking performances are presented in Fig. 6 . The value of the objective function becomes smaller than 0.01 between horizontal-axis values of 4.42 and 6.97 for $\tau=0.001$ and 4.34 and 7.11 for $\tau=0.0001$, and the width of horizontal-axis values spreads to realize robustness.

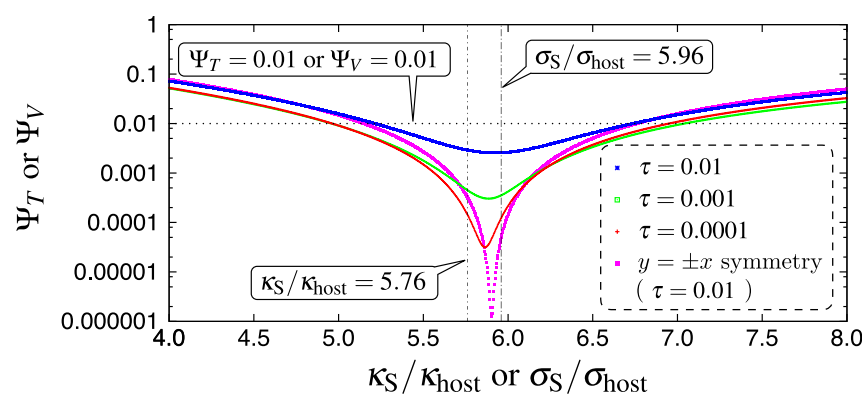

FIG. 5. Dependence of the cloaking performance on the relative conductivity. The optimal configurations are designed under $\kappa_{\mathrm{S}} / \kappa_{\text {host }}=386 / 67.0=5.76$ and $\sigma_{\mathrm{S}} / \sigma_{\text {host }}$ $=59.0 / 9.90=5.96$. 

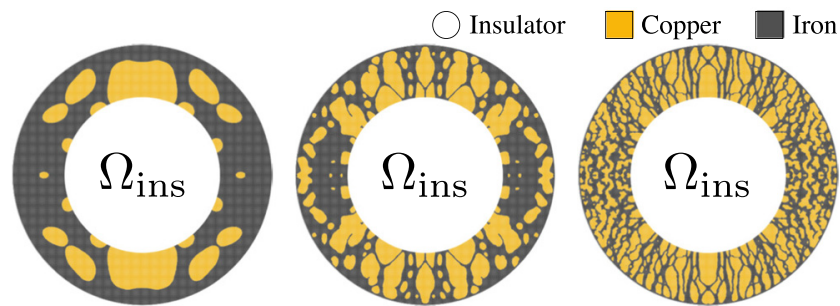

(a) $\tau=0.01, L_{\mathrm{p}}=9.55$
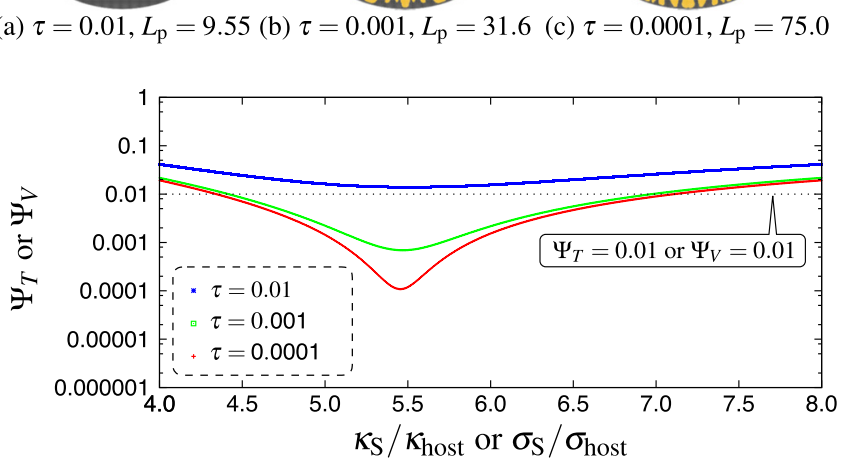

(d) $\Psi_{T}$ versus $\kappa_{\mathrm{S}} / \kappa_{\text {host }}$ or $\Psi_{V}$ versus $\sigma_{\mathrm{S}} / \sigma_{\text {host }}$

FIG. 6. Optimal configurations obtained by minimizing $F_{\max }$ for (a) $\tau=0.01$, (b) $\tau=0.001$, and (c) $\tau=0.0001$ and (d) dependence of the cloaking performance on the relative conductivity.

In summary, topology optimization incorporating both thermal conductivity and electrical direct current is established using an immersed boundary-level set method and CMA-ES, and a bifunctional cloak designed using the established methodology is presented. Optimal configurations composed of copper and iron restrain the disturbances of temperature and voltage distributions despite the presence of an insulator obstacle. To design a bifunctional cloak that operates on physical flows from multiple angles, we also demonstrate a topology optimization under structural symmetries about not only $x$ and $y$ axes but also $y= \pm x$. The optimal configuration almost has rotational symmetry, even though rotational symmetry is not imposed, and the configuration corresponds to the bilayer strategy previously proposed. Additionally, designs robust against a variation in material conductivities for bifunctional cloaking are demonstrated. The conductivity range restraining thermal and electrical disturbances smaller than $1 \%$ is spread to minimize the maximum of the multiple objective functions computed under several conductivities.
This work was supported by JSPS KAKENHI (Grant No. 17K17778). We thank Glenn Pennycook, MSc, from Edanz Group (www.edanzediting.com/ac) for editing a draft of this manuscript.

\section{REFERENCES}

${ }^{1}$ A. Alù and N. Engheta, Phys. Rev. E 72, 016623 (2005).

${ }^{2}$ U. Leonhardt, Science 312, 1777 (2006).

${ }^{3}$ J. B. Pendry, D. Schurig, and D. R. Smith, Science 312, 1780 (2006).

${ }^{4}$ S. A. Cummer and D. Schurig, New J. Phys. 9, 45 (2007).

${ }^{5}$ C. Z. Fan, Y. Gao, and J. P. Huang, Appl. Phys. Lett. 92, 251907 (2008).

${ }^{6}$ F. Yang, Z. L. Mei, T. Y. Jin, and T. J. Cui, Phys. Rev. Lett. 109, 053902 (2012).

${ }^{7}$ A. Alù and N. Engheta, Phys. Rev. Lett. 102, 233901 (2009).

${ }^{8}$ R. Fleury, J. Soric, and A. Alù, Phys. Rev. B 89, 045122 (2014).

${ }^{9}$ T. Han, X. Bai, J. T. L. Thong, B. Li, and C.-W. Qiu, Adv. Mater. 26, 1731 (2014).

${ }^{10}$ M. P. Bendsøe and N. Kikuchi, Comput. Methods Appl. Mech. Eng.. 71, 197 (1988).

${ }^{11}$ J. Andkjær and O. Sigmund, Appl. Phys. Lett. 98, 021112 (2011).

${ }^{12}$ J. Andkjær, N. A. Mortensen, and O. Sigmund, Appl. Phys. Lett. 100, 101106 (2012).

${ }^{13}$ G. Fujii, H. Watanabe, T. Yamada, T. Ueta, and M. Mizuno, Appl. Phys. Lett. 102, 251106 (2013).

${ }^{14}$ G. Fujii and T. Ueta, Phys. Rev. E 94, 043301 (2016).

${ }^{15}$ N. Kishimoto, K. Izui, S. Nishiwaki, and T. Yamada, Appl. Phys. Lett. 110, 201104 (2017).

${ }^{16}$ G. Fujii, M. Takahashi, and Y. Akimoto, Comput. Methods Appl. Mech. Eng. 332, 624 (2018).

${ }^{17}$ J. Andkjær and O. Sigmund, J. Vib. Acoust. 135, 041011 (2013).

${ }^{18}$ M. Takahashi, Y. Akimoto, and G. Fujii, Trans. Jpn. Soc. Mech. Eng. 84, 17-00590 (2018).

${ }^{19} \mathrm{G}$. Fujii, Y. Akimoto, and M. Takahashi, Appl, Phys. Lett. 112, 061108 (2018).

${ }^{20} \mathrm{G}$. Fujii and Y. Akimoto, Int. J. Heat Mass Transfer 137, 1312 (2019).

${ }^{21} \mathrm{G}$. Fujii, Y. Akimoto, and M. Takahashi, J. Appl. Phys. 123, 233102 (2018).

${ }^{22}$ G. Fujii and Y. Akimoto, Opt. Lett. 44, 2057 (2019).

${ }^{23}$ J. Y. Li, Y. Gao, and J. P. Huang, J. Appl. Phys. 108, 074504 (2010).

${ }^{24}$ M. Moccia, G. Castaldi, S. Savo, Y. Sato, and V. Galdi, Phys. Rev. X 4, 021025 (2014).

${ }^{25}$ Y. Ma, Y. Liu, M. Raza, Y. Wang, and S. He, Phys. Rev. Lett. 113, 205501 (2014).

${ }^{26}$ M. Raza, Y. Liu, and Y. Ma, J. Appl. Phys. 117, 024502 (2015).

${ }^{27}$ C. Lan, K. Bi, Z. Gao, B. Li, and J. Zhou, Appl. Phys. Lett. 109, 201903 (2016).

${ }^{28}$ T. Han, X. Bai, D. Gao, J. T. L. Thong, B. Li, and C.-W. Qiu, Phys. Rev. Lett. 112, 054302 (2014).

${ }^{29}$ T. Han, P. Yang, Y. Li, D. Lei, B. Li, K. Hippalgaonkar, and C.-W. Qiu, Adv. Mater. 30, 1804019 (2018).

${ }^{30}$ T. Han, H. Ye, Y. Luo, S. P. Yeo, J. Teng, S. Zhang, and C.-W. Qiu, Adv. Mater. 26, 3478 (2014).

${ }^{31}$ N. Hansen and A. Ostermeier, Evol. Comput. 9, 159 (2001).

${ }^{32}$ Y. Liu, W. Guo, and T. Han, Int. J. Heat Mass Transfer 115, 1 (2017).

${ }^{33}$ J. Qin, W. Luo, P. Yang, B. Wang, T. Deng, and T. Han, Int. J. Heat Mass Transfer 141, 487 (2019). 